\title{
Irish Veterinary Journal reviewer acknowledgement 2013
}

Michael L Doherty

\section{Contributing reviewers}

The Irish Veterinary Journal editorial team would like to thank the following colleagues who contributed to peer review for the journal in 2013.

Naveed Akhtar
Pakistan

Valeria Albanese

United States of America

Charles Bacon

United States of America

Marijke Beltman

Ireland

James Breen

United Kingdom

Ben Brilot

United Kingdom

Murray Brown

United States of America

Franz Brulisauer

United Kingdom

Bryce Buddle

New Zealand

Sean Callanan

Ireland

Joe Cassidy

Ireland

Stephanie Caston

United States of America

Peter Chapman

United States of America

$\begin{array}{ll}\text { Karin Darpel } & \text { Niel Karrow } \\ \text { United Kingdom } & \text { Canada } \\ \text { Theo de Waal } & \text { Lauren Lacorcia } \\ \text { Ireland } & \text { Australia } \\ \text { Michael Diskin } & \text { Desmond Leadon } \\ \text { Ireland } & \text { Ireland } \\ \text { Fiona Doohan } & \text { Ingrid Lorenz } \\ \text { Ireland } & \text { Ireland }\end{array}$

Edward Dubovi

United States of America

Keith Macmillan

Australia

\section{Seamus Fanning}

Ireland

James Gibbons

Ireland

John Gilmore

Ireland

Eamonn Gormley

Ireland

Jeanne Gradé

Belgium

Terence Grimes

Ireland

\section{John Madigan}

United States of America

Bryan Markey

Ireland

John Mee

Ireland

Patti Miller

United States of America

Stefano Nardelli

Italy

Peter Nettleton

United Kingdom

Kei Hayashi

United States of America

Erasmus Okine

Canada

Bernhard Kaltenboeck

United States of America

\section{Ronan O'Neill}

Ireland

Correspondence: michael.doherty@ucd.ie

UCD School of Veterinary Medicine, University College Dublin, Belfield,

Dublin 4, Ireland 


\section{Chris Oura}

Trinidad and Tobago

Felisbina Queiroga

Portugal

\section{Sarah Randolph}

United Kingdom

Charlotte Sandersen

Belgium

Horst Schirrmeier

Germany
Julie Settlage

United States of America

\section{Robert Shiel}

Australia

George Stewart

United States of America

David Suarez

United States of America

David Verner-Jeffreys

United Kingdom
Andrew Waller

United Kingdom

Keith Way

United Kingdom

Paul Whyte

Ireland

Mike Woods

Ireland

doi:10.1186/2046-0481-67-3

Cite this article as: Doherty: Irish Veterinary

Journal reviewer acknowledgement 2013.

Irish Veterinary Journal 2014 67:3. 